\title{
Immobilizing Pertechnetate in Ettringite via Sulfate Substitution
}

Sarah A. Saslow ${ }^{1 *}$, Sebastien N. Kerisit ${ }^{2}$, Tamas Varga ${ }^{3}$, Sebastian T. Mergelsberg ${ }^{2}$, Claire L.

Corkhill $^{4}$, Michelle M.V. Snyder ${ }^{1}$, Nancy M. Avalos ${ }^{1}$, Antonia S. Yorkshire ${ }^{4}$, Daniel J. Bailey ${ }^{4}$, Jarrod Crum $^{1}$, and R. Matthew Asmussen ${ }^{1}$.

${ }^{1}$ Energy and Environment Directorate, Pacific Northwest National Laboratory, Richland, WA 99352, USA

${ }^{2}$ Physical and Computational Sciences Directorate, Physical Sciences Division, Pacific Northwest National Laboratory, Richland, WA 99352, USA

${ }^{3}$ Environmental Molecular Sciences Laboratory, Earth and Biological Sciences Directorate, Pacific Northwest National Laboratory, Richland, WA 99352, USA

${ }^{4}$ NucleUS Immobilisation Science Laboratory, Department of Materials Science and Engineering, The University of Sheffield, Sheffield, U.K.

* Corresponding author: sarah.saslow@pnnl.gov, ORCID 0000-0002-8385-2676

Summary of Supporting Information Content

18 Pages

7 Figures

5 Tables 
Table S1. $\mathrm{TcO}_{4}{ }^{-}$Apparent Partition Coefficient and Associated Measured and Calculated Experimental Values

\begin{tabular}{|c|c|c|c|c|c|c|c|c|c|c|c|}
\hline $\begin{array}{c}\text { Sample } \\
\text { Vol. }\end{array}$ & $\begin{array}{c}{\left[\mathrm{TcO}_{4}^{-}\right]} \\
\text {initial }\end{array}$ & $\begin{array}{l}{\left[\mathrm{TcO}_{4}^{-}\right]} \\
\text {final }\end{array}$ & $\begin{array}{c}\mathrm{TcO}_{4}^{-} \\
\text {Removed }\end{array}$ & $\mathrm{TcO}_{4}^{-}$in solid ${ }^{\$}$ & $\begin{array}{c}{\left[\mathrm{SO}_{4}{ }^{2-}\right]} \\
\text { initial }\end{array}$ & $\begin{array}{c}{\left[\mathrm{SO}_{4}{ }^{2-}\right]} \\
\text { final }\end{array}$ & $\mathrm{SO}_{4}^{2-}$ in solid ${ }^{\$}$ & Final pH & $\begin{array}{c}\text { Total Solid } \\
\text { Mass }^{\wedge}\end{array}$ & $\begin{array}{c}\text { Ettringite } \\
\text { Mass }^{\#}\end{array}$ & $\begin{array}{c}\text { Apparent } \\
\text { Partition } \\
\text { Coefficient } \\
\left(K^{\prime} d\right) \%\end{array}$ \\
\hline $\mathrm{mL}$ & ppm & ppm & $\%$ & mol & ppm & $\mathrm{ppm}$ & mol & & $\mathrm{g}$ & $\mathrm{g}$ & $\times 10^{-3}$ \\
\hline 50.0 & 0.86 & $0.80 \pm 0.02$ & $6.3 \pm 1.8$ & $1.7 \times 10^{-8} \pm 0.5 \times 10^{-8}$ & 1515 & $23.0 \pm 2.3$ & $7.7 \times 10^{-4} \pm 1.2 \times 10^{-6}$ & $12.48 \pm 0.02$ & $0.236 \pm 0.073$ & $0.053 \pm 0.017$ & $1.0 \pm 0.4$ \\
\hline 50.0 & 8.2 & $8.1 \pm 0.2$ & $1.5 \pm 2.3$ & $3.9 \times 10^{-8} \pm 5.9 \times 10^{-8}$ & 1515 & $22.8 \pm 1.8$ & $7.7 \times 10^{-4} \pm 9.4 \times 10^{-7}$ & $12.49 \pm 0.00^{*}$ & $0.203 \pm 0.002$ & $0.070 \pm 0.001$ & $0.2 \pm 0.4$ \\
\hline 50.0 & 95 & $81 \pm 3$ & $14 \pm 3$ & $4.2 \times 10^{-6} \pm 0.9 \times 10^{-6}$ & 1515 & $22.2 \pm 0.1$ & $7.7 \times 10^{-4} \pm 5.2 \times 10^{-8}$ & $12.51 \pm 0.02$ & $0.156 \pm 0.013$ & $0.040 \pm 0.003$ & $2.5 \pm 0.6$ \\
\hline 20.2 & 166 & 154 & 7.1 & $1.5 \times 10^{-6}$ & 1500 & 21.0 & $3.1 \times 10^{-4}$ & 12.45 & 0.078 & 0.038 & 1.1 \\
\hline
\end{tabular}

$\$ \mathrm{TcO}_{4}^{-}$or $\mathrm{SO}_{4}{ }^{2-}$ in solid $(\mathrm{mol})=\left[\left(\left[\mathrm{TcO}_{4}{ }^{-}, \mathrm{SO}_{4}{ }^{2-}\right]\right.\right.$, initial - $\left[\mathrm{TcO}_{4}^{-}, \mathrm{SO}_{4}{ }^{2-}\right]$, final) $*($ Sample Vol. $\left.(\mathrm{mL}) / 1000(\mathrm{~mL} / \mathrm{L}))\right] / \mathrm{Molar} \mathrm{Mass} \mathrm{of} \mathrm{TcO}_{4}^{-}$or SO${ }_{4}{ }^{2-}(\mathrm{mg} / \mathrm{mol})$

${ }^{*}$ Represents the average and standard deviation of two out of three replicates. The third replicate was omitted due to a lower than normal $\mathrm{pH}$ measurement, $\mathrm{pH} 8.16$, and observed precipitates at the bottom of the collection vial. Precipitates were not observed in any of the other samples.

${ }^{\wedge}$ Average and standard deviation calculated from two of three replicates for each condition. The third replicate was sub-sampled for XRD and EXAFS analysis before a total sample mass could be recorded.

\# Mass of ettringite calculated based on the XRD quantification results presented in Table 1 for each set of conditions. These solids were also those used for EXAFS measurements.

${ }^{\%}$ Apparent Partition Coefficient, $K_{d}{ }_{d}=\left(\mathrm{TcO}_{4}{ }^{-}\right.$in solid $(\mathrm{mol}) / \mathrm{SO}_{4}{ }^{2-}$ in solid $\left.(\mathrm{mol})\right) /\left(\left[\mathrm{TcO}_{4}{ }^{-}\right]\right.$, final/ $\left[\mathrm{SO}_{4}{ }^{2-}\right]$, final $)$ 
Ettringite Pellet Synthesis and $\mathrm{TcO}_{4}$ C Contacting. $\mathrm{Ca}(\mathrm{OH})_{2}$ (ACS Reagent, $\geq 95.0 \%$, Sigma Aldrich) and $\mathrm{Al}_{2}\left(\mathrm{SO}_{4}\right)_{3} \cdot 16 \mathrm{H}_{2} \mathrm{O}$ (ACS Reagent, $\geq 95.0 \%$, Sigma Aldrich) were added to Milli-Q water $(18.2 \mathrm{M} \Omega)$ in stoichiometric amounts, according to the reaction outlined in Equation S1. The solution / suspension was mixed well before pouring into Teflon lined Parr vessels that were sealed, tightened, and placed into a heating block set to $150^{\circ} \mathrm{C}$ for 7 days.

$$
\mathrm{Ca}(\mathrm{OH})_{2}+\mathrm{Al}_{2}\left(\mathrm{SO}_{4}\right)_{3}+\mathrm{H}_{2} \mathrm{O}(\mathrm{xs}) \rightarrow \mathrm{Ca}_{6} \mathrm{Al}_{2}\left(\mathrm{SO}_{4}\right)_{3}(\mathrm{OH})_{12} \cdot 26 \mathrm{H}_{2} \mathrm{O} \quad \text { Eq. } \mathrm{S} 1
$$

After reaction, the resulting solid was dried at $35^{\circ} \mathrm{C}$ for 24 hours. The dried solids were stored under a $\mathrm{N}_{2}$ atmosphere to reduce carbonation prior to subsequent analyses. To prepare Tccontacted ettringite pellets, 25 - $49 \mathrm{mg}$ of the ettringite powder was pressed into a $6 \mathrm{~mm}$ pellet. Then, $20-39 \mu \mathrm{L}$ of the $10 \mathrm{mM} \mathrm{NH}_{4} \mathrm{TcO}_{4}$ solution (made in Ar degassed Milli-Q water) was dropped directly onto the pellet and left to soak through and dry for 24 hours. The pellet was then either secured within an aluminum EXAFS holder ladder using epoxy resin or powdered for XRD analysis. XRD measurements were performed on a D2 Bruker Phaser Desktop instrument over a $2 \theta$ range of $5-50^{\circ}$ with a 0.02 step size and 1 s counting time per step. The ettringite solid was prepared for measurement using a low background $\mathrm{Si}\left(\begin{array}{lll}1 & 1 & 1\end{array}\right)$ holder. The powder was compacted into the sample well using a glass slide to ensure the powder was flush with the top of the sample holder.

Gypsum Synthesis. $10 \mathrm{~mL}$ of $1 \mathrm{M} \mathrm{Na} 2 \mathrm{SO}_{4}$ solution made in DDI (Millipore $18.2 \mathrm{M} / \mathrm{cm}$ ) was spiked with $0.2 \mathrm{~mL}$ of a $10,000 \mathrm{ppm}{ }^{99} \mathrm{Tc}\left(\right.$ as $\left.\mathrm{NaTcO}_{4}\right)$ solution and then combined with $10 \mathrm{~mL}$ of $1 \mathrm{M} \mathrm{CaCl}_{2} \cdot 2 \mathrm{H}_{2} \mathrm{O}$ in a $50 \mathrm{~mL}$ centrifuge tube. Gypsum precipitation proceeded over a two-day period with occasional hand mixing and exposure to ambient conditions. After two days the solid was separated from the remaining solution using a $0.45 \mu \mathrm{m}$ sterile filter (Nalgene () ). Solids were allowed to dry in air before processing for solid phase characterization. For gypsum without Tc, the same procedure was used but with $0.1 \mathrm{M}$ solutions and without $\mathrm{NaTcO}_{4}$ spiking.

X-ray Diffraction (XRD) Sample Preparation: Prior to XRD analysis, each sample was homogenized with a rutile (NIST or Sigma Aldrich, CAS \# 224227) standard, added at $10 \mathrm{wt} \%$, using a mortar and pestle. Powdered samples were packed into zero background quartz sample holders or, if sample quantity was limited, mixed with analytical grade ethanol and allowed to dry as a homogeneous thin layer on the back side of the sample holder. Holders were analyzed within custom stages with Kapton windows to prevent dispersion of radiological sample material. 


\section{Epoxy-Embedded Powder Preparation Methods and Microprobe X-ray Fluorescence Mapping}

$(\boldsymbol{\mu X R F ) . ~ M i c r o p r o b e} \mathrm{X}$-ray fluorescence $(\mu \mathrm{XRF})$ measurements were performed on powdered solids secured in epoxy (EpoThin 2, Buehler Inc.) and contained with Kapton film. The final sample thickness was maintained to $\leq 25 \mu \mathrm{m}$ thick. At BL 10-2, a $\operatorname{Si}(111) \varphi=90^{\circ}$ double-crystal monochromator was used to select the incident energy with $\mathrm{N}_{2}$ filled ion chambers to monitor the flux. X-ray fluorescence was collected using a four-element Si-drift Vortex detector using Quantum Xspress3 pulse processing electronics. The monochromator was calibrated to the Mo absorption K-edge (20,000 keV) using Mo foil. $\mu$ XRF maps were collected at $21.2 \mathrm{keV}$ with a 100 $\mu \mathrm{m}$ beam size in $25 \mu \mathrm{m}$ steps with a dwell time of $25 \mathrm{msec}$ to identify locations rich in Tc. Element maps were analyzed and compiled using the MicroAnalysis Toolkit. ${ }^{1}$

High Energy X-ray Diffraction (HE-XRD) Pattern Refinement: Refinement of diffraction data was performed in GSAS-II using published crystal structures of ettringite, ${ }^{18}$ gypsum, ${ }^{19}$ and calcite. ${ }^{20}$ To subtract background signal, an empty region of the sample holder was measured independently. Refinement variables were restricted to unit cell parameters, phase concentration, preferred alignment (using a spherical harmonics correction of order 6), and particle size (for single/pure phases). Instrument-dependent peak broadening was refined using diffraction of a diluted $\mathrm{TiO}_{2}$ standard from NIST diffraction standard set 674a.

Ab initio Molecular Dynamics Simulations and EXAFS Fitting Details. Plane-wave density functional theory (DFT) calculations were performed with VASP (Vienna Ab-initio Simulation Package $)^{2-4}$ using the projector augmented-wave (PAW) approach ${ }^{5,6}$ and the generalized gradient approximation exchange-correlation functional of Perdew, Burke, and Ernzerhof (PBE) ${ }^{7,8}$ with Grimme dispersion corrections ${ }^{9}(\mathrm{G})$. The PBE PAW potentials were obtained from the VASP database for technetium (36), calcium (12), sulfur (10), aluminum (10), sodium (4), oxygen (2), and hydrogen (0) with the number of core electrons shown in parentheses.

Constant-pressure energy minimizations were first performed to determine the optimized crystal structure of ettringite and gypsum at the $\mathrm{PBE}+\mathrm{G}$ level of approximation. The calculated ettringite $\left(a=b=10.985 \AA(\Delta=-1.6 \%), c=21.381 \AA(\Delta=0.1 \%), \beta=118^{\circ}(\Delta=-1.5 \%)\right)$ and gypsum $(a$ $=5.676 \AA(\Delta=-0.1 \%), b=15.006 \AA(\Delta=-1.4 \%), c=6.490 \AA(\Delta=-0.6 \%), \beta=118^{\circ}(\Delta=$ $-0.5 \%$ ), space group I2/a) lattice parameters compared well to neutron powder diffraction data. ${ }^{10,11}$ The gypsum optimized cell was scaled to a $2 \times 1 \times 2$ supercell for the incorporation calculations 
whereas the ettringite was not scaled as already large with all lattice vectors at least $10 \AA$ in length. As a benchmark, a constant-pressure minimization of $\mathrm{NaTcO}_{4}$ was also performed and the optimized lattice parameters were in excellent agreement with experiment ${ }^{12}(a=b=5.339 \AA(\Delta=$ $0.1 \%), c=11.864 \AA(\Delta=0.1 \%))$.

For ettringite, three incorporation modes were investigated: (1) One $\mathrm{TcO}_{4}{ }^{-}$ion was substituted for two $\mathrm{H}_{2} \mathrm{O}$ molecules and a proton added to a neighboring $\mathrm{H}_{2} \mathrm{O}$ molecule (hence forming a hydronium ion) for charge compensation, two initial orientations of the $\mathrm{TcO}_{4}{ }^{-}$ion were considered; (2) One $\mathrm{TcO}_{4}{ }^{-}$ion was substituted for $\mathrm{SO}_{4}{ }^{2-}$ ion and a proton removed from a neighboring $\mathrm{H}_{2} \mathrm{O}$ molecule for charge compensation, all three $\mathrm{SO}_{4}{ }^{2-}$ crystallographic positions were considered; and (3) One $\mathrm{TcO}_{4}{ }^{-}$ion was substituted for two $\mathrm{H}_{2} \mathrm{O}$ molecules and one $\mathrm{TcO}_{4}{ }^{-}$ion was substituted for a $\mathrm{SO}_{4}{ }^{2-}$, the case where the two $\mathrm{TcO}_{4}{ }^{-}$ions are in nearest-neighbor positions and the case where they are in a distant position were considered. For gypsum, two incorporation modes were investigated: (1) One $\mathrm{TcO}_{4}{ }^{-}$ion was substituted for a $\mathrm{SO}_{4}{ }^{2-}$ ion and a proton removed from a neighboring $\mathrm{H}_{2} \mathrm{O}$ molecule for charge compensation, two crystallographic positions from which to remove the proton were considered; and (2) $\mathrm{One}_{\mathrm{TcO}_{4}}{ }^{-}$ion was substituted for a $\mathrm{SO}_{4}{ }^{2-}$ ion and a neighboring $\mathrm{Ca}^{2+}$ ion was substituted by a $\mathrm{Na}^{+}$ion for charge compensation, two positions for the cation substitution were considered. These reaction pathways are summarized in Table S2. 
Table S2. AIMD Trajectories for $\mathrm{TcO}_{4}{ }^{-}$Substitution into Ettringite and Gypsum.

\begin{tabular}{|c|c|c|c|}
\hline Label & Note & Configuration & Net reaction \\
\hline \multicolumn{4}{|c|}{ Ettringite } \\
\hline $\mathrm{H}_{2} \mathrm{O}-1$ & up & Ettringite $+\mathrm{TcO}_{4}^{-}+\mathrm{H}^{+}-2 \mathrm{H}_{2} \mathrm{O}$ & $3 \mathrm{H}_{2} \mathrm{O} \rightarrow \mathrm{TcO}_{4}^{-}+\mathrm{H}_{3} \mathrm{O}^{+}$ \\
\hline $\mathrm{H}_{2} \mathrm{O}-2$ & down & Ettringite $+\mathrm{TcO}_{4}^{-}+\mathrm{H}^{+}-2 \mathrm{H}_{2} \mathrm{O}$ & $3 \mathrm{H}_{2} \mathrm{O} \rightarrow \mathrm{TcO}_{4}^{-}+\mathrm{H}_{3} \mathrm{O}^{+}$ \\
\hline $\mathrm{SO}_{4}-1$ & crystal position 1 & Ettringite $+\mathrm{TcO}_{4}^{-}-\mathrm{SO}_{4}^{2-}-\mathrm{H}^{+}$ & $\mathrm{SO}_{4}^{2-}+\mathrm{H}_{2} \mathrm{O} \rightarrow \mathrm{TcO}_{4}^{-}+\mathrm{OH}^{-}$ \\
\hline $\mathrm{SO}_{4}-2 \mathrm{a}^{*}$ & crystal position 2 & Ettringite $+\mathrm{TcO}_{4}^{-}-\mathrm{SO}_{4}{ }^{2-}-\mathrm{H}^{+}$ & $\mathrm{SO}_{4}^{2-}+\mathrm{H}_{2} \mathrm{O} \rightarrow \mathrm{TcO}_{4}^{-}+\mathrm{OH}^{-}$ \\
\hline $\mathrm{SO}_{4}-2 \mathrm{~b}^{*}$ & $\begin{array}{l}\text { crystal position } 2, \\
\text { bound directly to } \mathrm{Ca}^{2+}\end{array}$ & Ettringite $+\mathrm{TcO}_{4}^{-}-\mathrm{SO}_{4}{ }^{2-}-\mathrm{H}^{+}$ & $\mathrm{SO}_{4}{ }^{2-}+\mathrm{H}_{2} \mathrm{O} \rightarrow \mathrm{TcO}_{4}^{-}+\mathrm{OH}^{-}$ \\
\hline $\mathrm{SO}_{4}-3$ & crystal position 3 & Ettringite $+\mathrm{TcO}_{4}^{-}-\mathrm{SO}_{4}^{2-}-\mathrm{H}^{+}$ & $\mathrm{SO}_{4}^{2-}+\mathrm{H}_{2} \mathrm{O} \rightarrow \mathrm{TcO}_{4}^{-}+\mathrm{OH}^{-}$ \\
\hline $\mathrm{H}_{2} \mathrm{O}-\mathrm{SO}_{4}-1$ & nearest neighbor & Ettringite $+2 \mathrm{TcO}_{4}^{-}-\mathrm{SO}_{4}{ }^{2-}-2 \mathrm{H}_{2} \mathrm{O}$ & $\mathrm{SO}_{4}^{2-}+2 \mathrm{H}_{2} \mathrm{O} \rightarrow 2 \mathrm{TcO}_{4}^{-}$ \\
\hline $\mathrm{H}_{2} \mathrm{O}-\mathrm{SO}_{4}-2$ & distant & Ettringite $+2 \mathrm{TcO}_{4}^{-}-\mathrm{SO}_{4}{ }^{2-}-2 \mathrm{H}_{2} \mathrm{O}$ & $\mathrm{SO}_{4}^{2-}+2 \mathrm{H}_{2} \mathrm{O} \rightarrow 2 \mathrm{TcO}_{4}^{-}$ \\
\hline \multicolumn{4}{|c|}{ Gypsum } \\
\hline $\mathrm{SO}_{4}-\mathrm{H} 1$ & position 1 & Gypsum $+\mathrm{TcO}_{4}^{-}-\mathrm{SO}_{4}^{2-}-\mathrm{H}^{+}$ & $\mathrm{SO}_{4}^{2-}+\mathrm{H}_{2} \mathrm{O} \rightarrow \mathrm{TcO}_{4}^{-}+\mathrm{OH}^{-}$ \\
\hline $\mathrm{SO}_{4}-\mathrm{H} 2$ & position 2 & Gypsum $+\mathrm{TcO}_{4}^{-}-\mathrm{SO}_{4}{ }^{2-}-\mathrm{H}^{+}$ & $\mathrm{SO}_{4}^{2-}+\mathrm{H}_{2} \mathrm{O} \rightarrow \mathrm{TcO}_{4}^{-}+\mathrm{OH}^{-}$ \\
\hline $\mathrm{SO}_{4}-\mathrm{Na1}$ & position 1 & Gypsum $+\mathrm{TcO}_{4}^{-}+\mathrm{Na}^{+}-\mathrm{SO}_{4}{ }^{2-}-\mathrm{Ca}^{2+}$ & $\mathrm{SO}_{4}^{2-}+\mathrm{Ca}^{2+} \rightarrow \mathrm{TcO}_{4}^{-}+\mathrm{Na}^{+}$ \\
\hline $\mathrm{SO}_{4}-\mathrm{Na} 2$ & position 2 & Gypsum $+\mathrm{TcO}_{4}^{-}+\mathrm{Na}^{+}-\mathrm{SO}_{4}{ }^{2-}-\mathrm{Ca}^{2+}$ & $\mathrm{SO}_{4}^{2-}+\mathrm{Ca}^{2+} \rightarrow \mathrm{TcO}_{4}^{-}+\mathrm{Na}^{+}$ \\
\hline
\end{tabular}

The convergence criterion for the electronic self-consistent calculation was $10^{-5} \mathrm{eV}$ throughout. For the constant-pressure energy minimizations (ionic positions, cell volume, and cell shape are allowed to relax) of the pure unit cells and constant-volume energy minimizations (only the ionic positions are allowed to relax) of the substituted supercells, convergence was reached when the force on any atom was less than $0.01 \mathrm{eV} / \AA$. The plane-wave cutoff energy was $650 \mathrm{eV}$ in these calculations. $3 \times 1 \times 3$ and $5 \times 5 \times 3 k$-point meshes were used for the gypsum and $\mathrm{NaTcO}_{4}$ unit cells whereas $\Gamma$-point calculations $(1 \times 1 \times 1 k$-point mesh) were performed for the ettringite unit cell and all supercells.

NVT (constant number of particles, constant volume, and constant temperature) AIMD simulations were performed at $0 \mathrm{~K}, 15 \mathrm{~K}$, and $298 \mathrm{~K}$ for all the incorporation modes described above starting from the energy-minimized structures. All the AIMD simulations used a slightly reduced plane-wave cutoff energy of $600 \mathrm{eV}$ and were performed at the $\Gamma$ point with a $0.5 \mathrm{fs}$ integration time step. The AIMD simulations were run for $7 \mathrm{ps}$ whereby the atomic velocities were scaled to the target temperature every 12.5 fs for the first picosecond of simulation. Configurations were collected form each simulation at $50 \mathrm{fs}$ intervals for the last $5 \mathrm{ps}$ to generate a pool of 100 configurations.

For each configuration, a cluster with a radius of $5.5 \AA$ centered on the technetium atom was then generated to calculate all scattering paths with effective distances less than the cluster radius for a 
K-core hole of the central atom using FEFF9. ${ }^{13-15}$ For each incorporation mode, the spectra of all configurations were averaged to produce a group of standards to be used in the linear combination fits. The Fourier transform (FT) was applied to the averaged EXAFS spectra using IFEFFIT. ${ }^{16}$
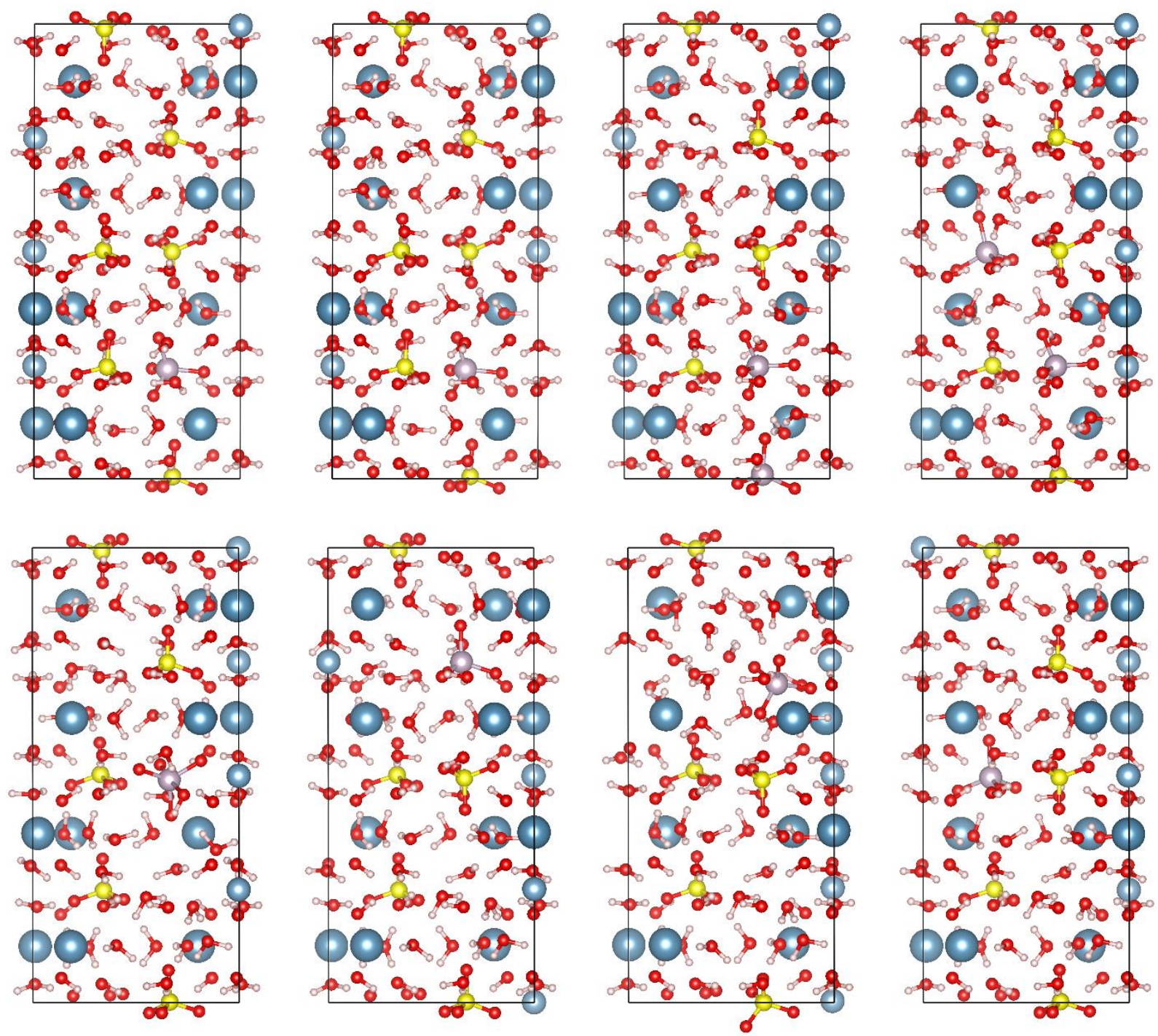

Figure S1. Ball-and-Stick Atomistic Models of $\mathrm{TcO}_{4}^{-}$Incorporated in Ettringite. All models are from energy-minimized structures. (top row, left to right) Incorporation modes $\mathrm{H}_{2} \mathrm{O}-$ 1, $\mathrm{H}_{2} \mathrm{O}-2, \mathrm{H}_{2} \mathrm{O}-\mathrm{SO}_{4}-1$, and $\mathrm{H}_{2} \mathrm{O}-\mathrm{SO}_{4}-2$. (bottom row, left to right) Incorporation modes $\mathrm{SO}_{4}-1$, $\mathrm{SO}_{4}-2 \mathrm{a}, \mathrm{SO}_{4}-2 \mathrm{~b}$, and $\mathrm{SO}_{4}-3$. Tc, $\mathrm{S}, \mathrm{Ca}, \mathrm{Al}, \mathrm{O}$, and $\mathrm{H}$ atoms are shown as gray, yellow, large blue, small blue, red, and white balls, respectively. 

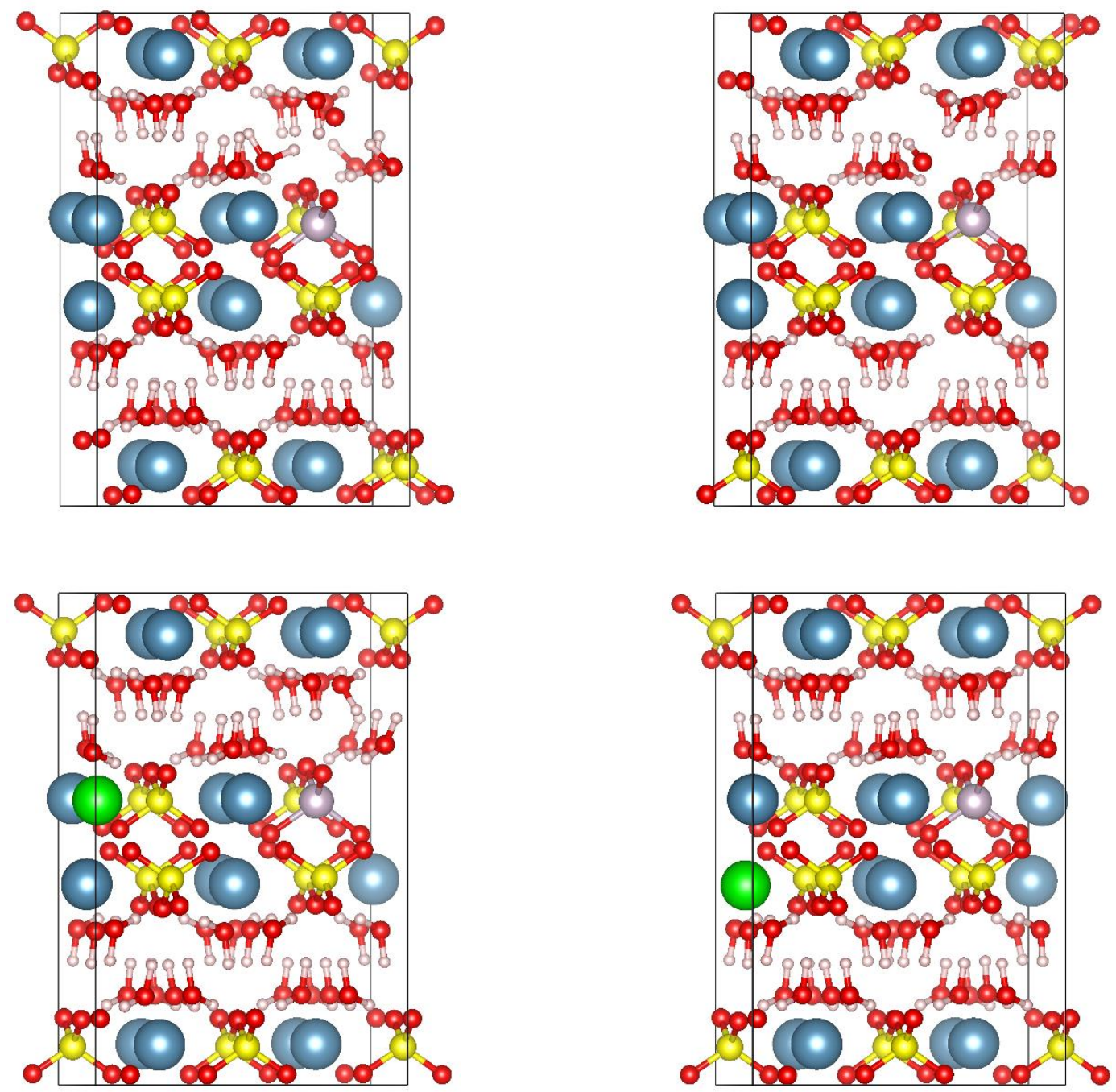

Figure S2. Ball-and-Stick Atomistic Models of $\mathrm{TcO}_{4}^{-}$Incorporated in Gypsum. All models are from energy-minimized structures. (top row, left to right) Incorporation modes $\mathrm{SO}_{4}-\mathrm{H} 1$ and $\mathrm{SO}_{4}-\mathrm{H} 2$. (bottom row, left to right) Incorporation modes $\mathrm{SO}_{4}-\mathrm{Na} 1$ and $\mathrm{SO}_{4}-\mathrm{Na} 2$. Tc, $\mathrm{S}, \mathrm{Ca}, \mathrm{Na}$, $\mathrm{O}$, and $\mathrm{H}$ atoms are shown as gray, yellow, blue, green, red, and white balls, respectively. 

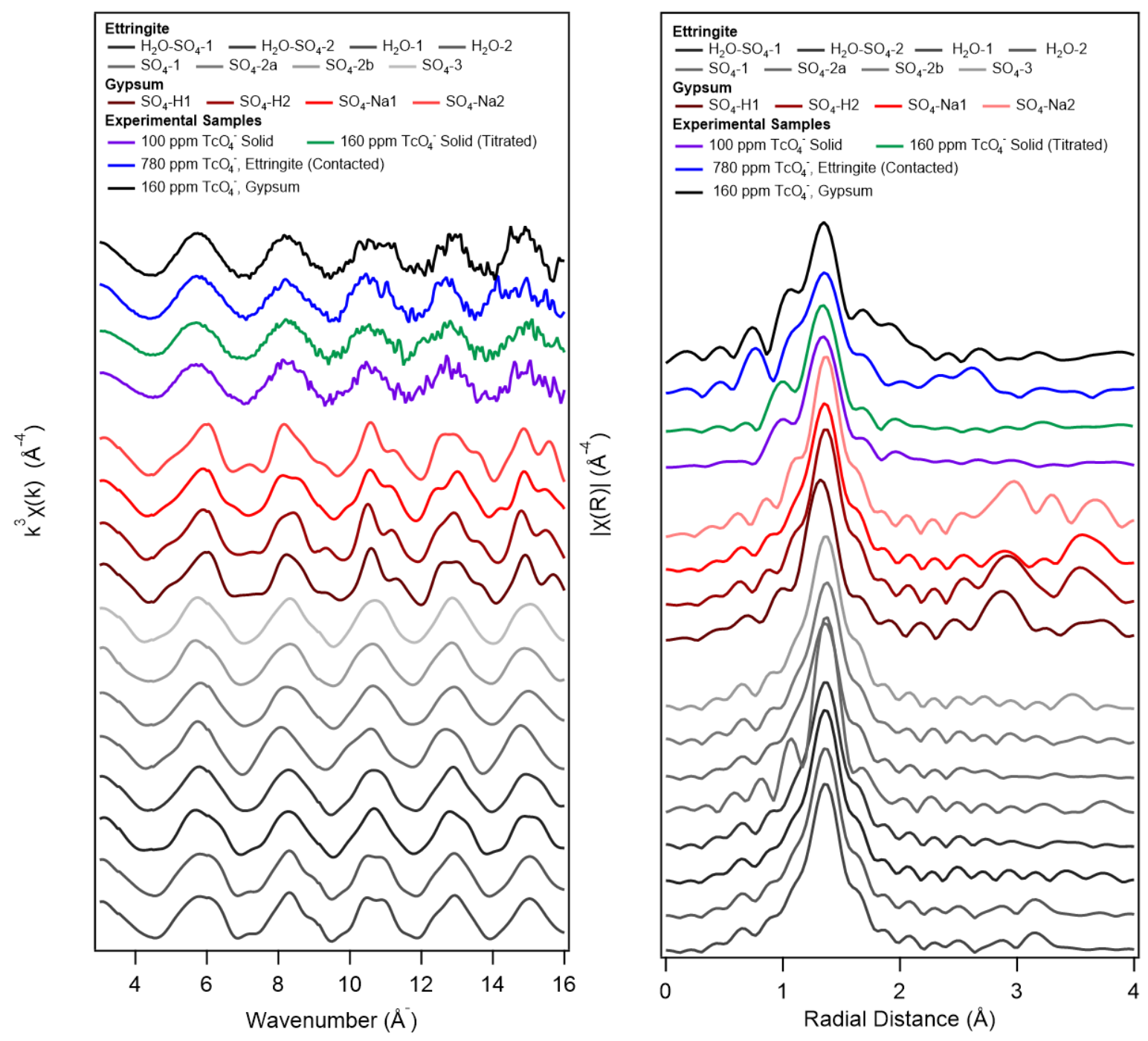

Figure S3. Experimental and AIMD Calculated Standard EXAFS Spectra for $\mathrm{TcO}_{4}{ }^{-}$Incorporated Solids. Tc K-edge EXAFS spectra (left) and their Fourier transforms (right) for select Tc-containing samples and AIMD calculated standards. Sample spectra were collected at 15 Kelvin. All PBE AIMD spectra were calculated assuming 15 Kelvin conditions. 

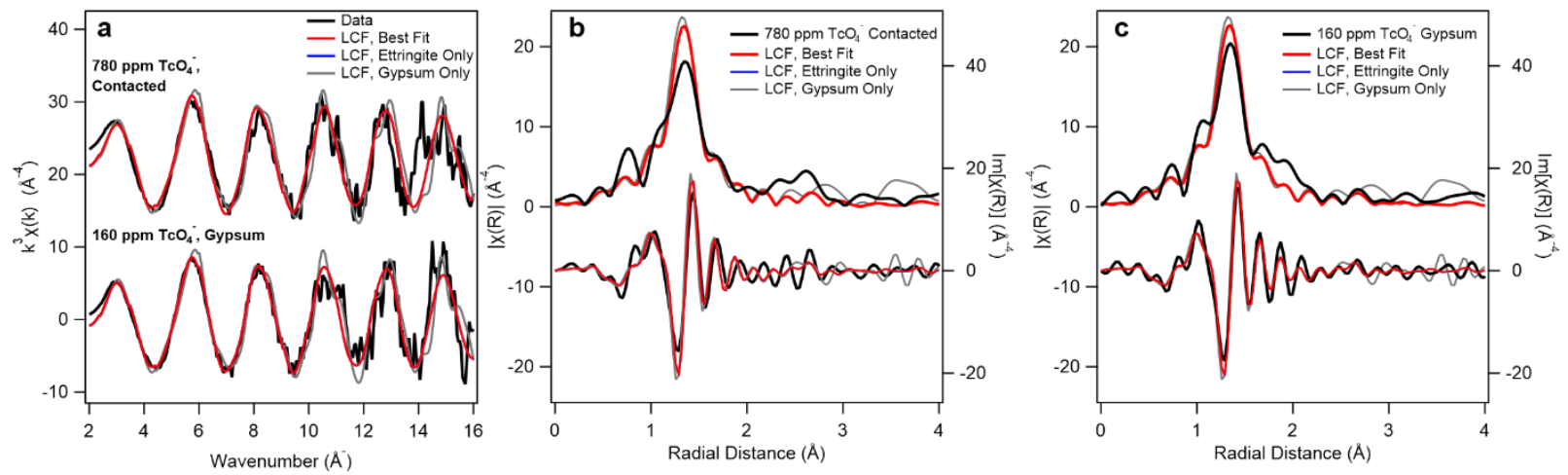

Figure S4. Structural environment of $\mathrm{TcO}_{4}{ }^{-}$and proposed incorporation modes by EXAFS and AIMD spectra. (a) Linear combination fits ( $k^{3}$ weight; best fit, red; ettringite-only, blue; and gypsum-only, grey) to the experimental Tc K-edge EXAFS spectra (black) collected at $15 \mathrm{~K}$ for ettringite contacted with $780 \mathrm{ppm} \mathrm{TcO}_{4}{ }^{-}$and gypsum precipitated in the presence of $160 \mathrm{ppm}$ $\mathrm{TcO}_{4}^{-}$. (b, c) Fourier transforms (magnitude, left axis; imaginary part, right axis) to the experimental EXAFS data shown in (A) and the best (red), ettringite-only (blue), and gypsumonly (grey) LCFs. 
Table S3. All LCFs to Experimental EXAFS Spectra Using AIMD Calculated Standard Spectra.

\begin{tabular}{|c|c|c|c|c|c|c|c|c|c|c|c|c|c|c|c|}
\hline \multicolumn{4}{|c|}{ Ettringite $+100 \mathrm{ppm} \mathrm{TcO}_{4}^{-}$} & \multicolumn{8}{|c|}{ Ettringite } & \multicolumn{4}{|c|}{ Gypsum } \\
\hline LCF & R Factor & $\chi^{2}$ & Red. $\chi^{2}$ & $\mathrm{H}_{2} \mathrm{O}-1$ & $\mathrm{H}_{2} \mathrm{O}-2$ & $\begin{array}{c}\mathrm{H}_{2} \mathrm{O}-\mathrm{SO}_{4}- \\
1\end{array}$ & $\begin{array}{c}\mathrm{H}_{2} \mathrm{O}-\mathrm{SO}_{4}- \\
2\end{array}$ & $\mathrm{SO}_{4-1}$ & $\mathrm{SO}_{4}-2 \mathrm{a}$ & $\mathrm{SO}_{4-2 b}$ & $\mathrm{SO}_{4-3}$ & $\mathrm{SO}_{4}-\mathrm{H1}$ & SO4-H2 & $\begin{array}{l}\mathrm{SO}_{4}- \\
\text { Na1 }\end{array}$ & $\begin{array}{l}\mathrm{SO}_{4}- \\
\mathrm{Na} 2\end{array}$ \\
\hline \multicolumn{16}{|c|}{ Ettringite Pathways Only } \\
\hline 1 & 0.25238 & 1226.88 & 4.72 & 0.00 & 1.00 & - & - & - & - & - & - & - & - & - & - \\
\hline 2 & 0.22343 & 1086.16 & 4.18 & - & - & 0.11 & 0.89 & - & - & - & - & - & - & - & - \\
\hline 3 & 0.17142 & 833.31 & 3.21 & - & - & - & - & 0.19 & 0.81 & 0.00 & - & - & - & - & - \\
\hline 4 & 0.17142 & 833.31 & 3.21 & - & - & - & - & 0.32 & - & - & 0.68 & - & - & - & - \\
\hline 5 & 0.17142 & 833.31 & 3.21 & - & - & - & - & 0.19 & 0.81 & 0.00 & 0.00 & - & - & - & - \\
\hline 6 & 0.17142 & 833.31 & 3.21 & - & - & 0.00 & 0.00 & 0.19 & 0.81 & 0.00 & 0.00 & - & - & - & - \\
\hline 7 & 0.17142 & 833.31 & 3.21 & 0.00 & 0.00 & 0.00 & 0.00 & 0.19 & 0.81 & 0.00 & 0.00 & - & - & - & - \\
\hline \multicolumn{16}{|c|}{ Gypsum Pathways Only } \\
\hline 8 & 0.37280 & 1812.29 & 6.97 & - & - & - & - & - & - & - & - & 0.66 & 0.34 & - & - \\
\hline 9 & 0.31855 & 1548.60 & 5.96 & - & - & - & - & - & - & - & - & - & - & 0.91 & 0.09 \\
\hline 10 & 0.31142 & 1513.90 & 5.87 & - & - & - & - & - & - & - & - & 0.23 & 0.12 & 0.65 & 0.00 \\
\hline \multicolumn{16}{|c|}{ Ettringite and Gypsum Pathways Considered } \\
\hline 11 & 0.17142 & 833.31 & 3.21 & 0.00 & 0.00 & 0.00 & 0.00 & 0.19 & 0.81 & 0.00 & 0.00 & 0.00 & 0.00 & 0.00 & 0.00 \\
\hline \multicolumn{16}{|c|}{$\mathrm{TcO}_{4}^{-}(\mathrm{g})$ Only } \\
\hline 12 & 0.24116 & 1172.38 & 4.51 & - & - & - & - & - & - & - & - & - & - & - & - \\
\hline
\end{tabular}

\begin{tabular}{|c|c|c|c|c|c|c|c|c|c|c|c|c|c|c|c|}
\hline \multicolumn{4}{|c|}{ Ettringite + 160 ppm TcO4 $4^{-}$} & \multicolumn{8}{|c|}{ Ettringite } & \multicolumn{4}{|c|}{ Gypsum } \\
\hline LCF & R Factor & $\chi^{2}$ & Red. $\chi^{2}$ & $\mathrm{H}_{2} \mathrm{O}-1$ & $\mathrm{H}_{2} \mathrm{O}-2$ & $\begin{array}{c}\mathrm{H}_{2} \mathrm{O}-\mathrm{SO}_{4}- \\
1\end{array}$ & $\begin{array}{c}\mathrm{H}_{2} \mathrm{O}-\mathrm{SO}_{4}- \\
2\end{array}$ & $\mathrm{SO}_{4-1}$ & $\mathrm{SO}_{4}-2 \mathrm{a}$ & $\mathrm{SO}_{4-2 b}$ & $\mathrm{SO}_{4-3}$ & $\mathrm{SO}_{4-\mathrm{H1}}$ & $\mathrm{SO}_{4}-\mathrm{H} 2$ & $\begin{array}{l}\mathrm{SO}_{4-} \\
\mathrm{Na1}\end{array}$ & $\begin{array}{l}\mathrm{SO}_{4-} \\
\mathrm{Na} 2\end{array}$ \\
\hline \multicolumn{16}{|c|}{ Ettringite Pathways Only } \\
\hline 1 & 0.28002 & 1214.50 & 4.67 & 0.00 & 1.00 & - & - & - & - & - & - & - & - & - & - \\
\hline 2 & 0.26041 & 1129.44 & 4.34 & - & - & 0.00 & 1.00 & - & - & - & - & - & - & - & - \\
\hline 3 & 0.19424 & 842.44 & 3.24 & - & - & - & - & 0.16 & 0.84 & 0.00 & - & - & - & - & - \\
\hline 4 & 0.26139 & 1133.66 & 4.36 & - & - & - & - & 0.32 & - & - & 0.68 & - & - & - & - \\
\hline 5 & 0.19424 & 842.44 & 3.24 & - & - & - & - & 0.16 & 0.84 & 0.00 & 0.00 & - & - & - & - \\
\hline 6 & 0.19424 & 842.44 & 3.24 & - & - & 0.00 & 0.00 & 0.16 & 0.84 & 0.00 & 0.00 & - & - & - & - \\
\hline 7 & 0.19424 & 842.44 & 3.24 & 0.00 & 0.00 & 0.00 & 0.00 & 0.16 & 0.84 & 0.00 & 0.00 & - & - & - & - \\
\hline \multicolumn{16}{|c|}{ Gypsum Pathways Only } \\
\hline 8 & 0.47602 & 2064.53 & 7.94 & - & - & - & - & - & - & - & - & 0.67 & 0.33 & - & - \\
\hline 9 & 0.36716 & 1592.43 & 6.12 & - & - & - & - & - & - & - & - & - & - & 1.00 & 0.00 \\
\hline 10 & 0.36519 & 1583.86 & 6.14 & - & - & - & - & - & - & - & - & 0.11 & 0.00 & 0.89 & 0.00 \\
\hline \multicolumn{16}{|c|}{ Ettringite and Gypsum Pathways Considered } \\
\hline 11 & 0.19424 & 842.44 & 3.24 & 0.00 & 0.00 & 0.00 & 0.00 & 0.16 & 0.84 & 0.00 & 0.00 & 0.00 & 0.00 & 0.00 & 0.00 \\
\hline \multicolumn{16}{|c|}{$\mathrm{TcO}_{4}^{-}(\mathrm{g})$ Only } \\
\hline 12 & 0.28766 & 1247.63 & 4.80 & - & - & - & - & - & - & - & - & - & - & - & - \\
\hline
\end{tabular}

Page S11 


\begin{tabular}{|c|c|c|c|c|c|c|c|c|c|c|c|c|c|c|c|}
\hline \multicolumn{4}{|c|}{780 ppm $\mathrm{TcO}_{4}^{-}$, Ettringite Pellet } & \multicolumn{8}{|c|}{ Ettringite } & \multicolumn{4}{|c|}{ Gypsum } \\
\hline LCF & R Factor & $\chi^{2}$ & $\begin{array}{c}\text { Red. } \\
\chi^{2}\end{array}$ & $\mathrm{H}_{2} \mathrm{O}-1$ & $\mathrm{H}_{2} \mathrm{O}-2$ & $\begin{array}{c}\mathrm{H}_{2} \mathrm{O}-\mathrm{SO}_{4}- \\
1\end{array}$ & \begin{tabular}{|c|}
$\mathrm{H}_{2} \mathrm{O}-\mathrm{SO}_{4}-$ \\
2
\end{tabular} & $\mathrm{SO}_{4-1}$ & $\mathrm{SO}_{4-2 a}$ & $\mathrm{SO}_{4-2 b}$ & $\mathrm{SO}_{4-3}$ & $\mathrm{SO}_{4}-\mathrm{H1}$ & $\mathrm{SO}_{4}-\mathrm{H} 2$ & $\begin{array}{l}\mathrm{SO}_{4-} \\
\mathrm{Na1}\end{array}$ & $\begin{array}{l}\mathrm{SO}_{4-} \\
\mathrm{Na}^{2}\end{array}$ \\
\hline \multicolumn{16}{|c|}{ Ettringite Pathways Only } \\
\hline 1 & 0.35510 & 2079.77 & 8.00 & 1.00 & 0.00 & - & - & - & - & - & - & - & - & - & - \\
\hline 2 & 0.33545 & 1964.64 & 7.56 & - & - & 0.00 & 1.00 & - & - & - & - & - & - & - & - \\
\hline 3 & 0.25790 & 1510.44 & 5.81 & - & - & - & - & 0.32 & 0.68 & 0.00 & - & - & - & - & - \\
\hline 4 & 0.30112 & 1763.59 & 6.78 & - & - & - & - & 0.48 & - & - & 0.52 & - & - & - & - \\
\hline 5 & 0.25790 & 1510.44 & 5.81 & - & - & - & - & 0.32 & 0.68 & 0.00 & 0.00 & - & - & - & - \\
\hline 6 & 0.25790 & 1510.44 & 5.81 & - & - & 0.00 & 0.00 & 0.32 & 0.68 & 0.00 & 0.00 & - & - & - & - \\
\hline 7 & 0.25790 & 1510.44 & 5.81 & 0.00 & 0.00 & 0.00 & 0.00 & 0.32 & 0.68 & 0.00 & 0.00 & - & - & - & - \\
\hline \multicolumn{16}{|c|}{ Gypsum Pathways Only } \\
\hline 8 & 0.47016 & 2753.65 & 10.59 & - & - & - & - & - & - & - & - & 0.63 & 0.37 & - & - \\
\hline 9 & 0.40677 & 2382.35 & 9.16 & - & - & - & - & - & - & - & - & - & - & 0.90 & 0.10 \\
\hline 10 & 0.40448 & 2368.95 & 9.11 & - & - & - & - & - & - & - & - & 0.18 & 0.00 & 0.82 & 0.00 \\
\hline \multicolumn{16}{|c|}{ Ettringite and Gypsum Pathways Considered } \\
\hline 11 & 0.25790 & 1510.44 & 5.81 & 0.00 & 0.00 & 0.00 & 0.00 & 0.32 & 0.68 & 0.00 & 0.00 & 0.00 & 0.00 & 0.00 & 0.00 \\
\hline \multicolumn{16}{|c|}{$\mathrm{TcO}_{4}^{-}(\mathrm{g})$ Only } \\
\hline 12 & 0.31527 & 1846.49 & 7.10 & - & - & - & - & - & - & - & - & - & - & - & - \\
\hline
\end{tabular}

\begin{tabular}{|c|c|c|c|c|c|c|c|c|c|c|c|c|c|c|c|}
\hline \multicolumn{4}{|c|}{160 ppm $\mathrm{TcO}_{4}^{-}, \mathrm{Gypsum}^{-}$} & \multicolumn{8}{|c|}{ Ettringite } & \multicolumn{4}{|c|}{ Gypsum } \\
\hline LCF & R Factor & $\chi^{2}$ & Red. $\chi^{2}$ & $\mathrm{H}_{2} \mathrm{O}-1$ & $\mathrm{H}_{2} \mathrm{O}-2$ & \begin{tabular}{|c}
$\mathrm{H}_{2} \mathrm{O}-\mathrm{SO}_{4}-$ \\
1 \\
\end{tabular} & \begin{tabular}{|c}
$\mathrm{H}_{2} \mathrm{O}-\mathrm{SO}_{4}-$ \\
2 \\
\end{tabular} & $\mathrm{SO}_{4-1}$ & $\mathrm{SO}_{4}-2 \mathrm{a}$ & $\mathrm{SO}_{4-2 b}$ & $\mathrm{SO}_{4-3}$ & $\mathrm{SO}_{4}-\mathrm{H1}$ & $\mathrm{SO}_{4}-\mathrm{H} 2$ & $\begin{array}{l}\mathrm{SO}_{4}- \\
\mathrm{Na1} \\
\end{array}$ & $\begin{array}{l}\mathrm{SO}_{4}- \\
\mathrm{Na2} \\
\end{array}$ \\
\hline \multicolumn{16}{|c|}{ Ettringite Pathways Only } \\
\hline 1 & 0.18749 & 1219.01 & 4.69 & 0.00 & 1.00 & - & - & - & - & - & - & - & - & - & - \\
\hline 2 & 0.17554 & 1141.32 & 4.39 & - & - & 0.00 & 1.00 & - & - & - & - & - & - & - & - \\
\hline 3 & 0.15329 & 996.61 & 3.85 & - & - & - & - & 0.20 & 0.80 & 0.00 & - & - & - & - & - \\
\hline 4 & 0.17191 & 1117.67 & 4.30 & - & - & - & - & 0.28 & - & - & 0.72 & - & - & - & - \\
\hline 5 & 0.15329 & 996.61 & 3.85 & - & - & - & - & 0.20 & 0.80 & 0.00 & 0.00 & - & - & - & - \\
\hline 6 & 0.15329 & 996.61 & 3.85 & - & - & 0.00 & 0.00 & 0.20 & 0.80 & 0.00 & 0.00 & - & - & - & - \\
\hline 7 & 0.15221 & 989.63 & 3.82 & 0.19 & 0.00 & 0.00 & 0.00 & 0.22 & 0.59 & 0.00 & 0.00 & - & - & - & - \\
\hline \multicolumn{16}{|c|}{ Gypsum Pathways Only } \\
\hline 8 & 0.30855 & 2006.08 & 7.72 & - & - & - & - & - & - & - & - & 0.51 & 0.49 & - & - \\
\hline 9 & 0.25407 & 1651.91 & 6.35 & - & - & - & - & - & - & - & - & - & - & 1.00 & 0.00 \\
\hline 10 & 0.25114 & 1632.83 & 6.28 & - & - & - & - & - & - & - & - & 0.16 & 0.00 & 0.84 & 0.00 \\
\hline \multicolumn{16}{|c|}{ Ettringite and Gypsum Pathways Considered } \\
\hline 11 & 0.17477 & 1136.31 & 4.39 & 0.00 & 0.18 & 0.00 & 0.80 & - & - & - & - & 0.02 & 0.00 & 0.00 & 0.00 \\
\hline 12 & 0.15221 & 989.63 & 3.82 & 0.19 & 0.00 & 0.00 & 0.00 & 0.22 & 0.59 & 0.00 & 0.00 & 0.00 & 0.00 & 0.00 & 0.00 \\
\hline \multicolumn{16}{|c|}{$\mathrm{TcO}_{4}^{-}(\mathrm{g})$ Only } \\
\hline 13 & 0.18927 & 1230.54 & 4.73 & - & - & - & - & - & - & - & - & - & - & - & - \\
\hline
\end{tabular}

Page S12 
Table S4. Energetics for $\mathrm{TcO}_{4}{ }^{-}$Substitution for $\mathrm{SO}_{4}{ }^{2-}$ Sites in Ettringite and Gypsum at Different Temperatures

\begin{tabular}{|c|c|c|c|c|c|c|c|}
\hline \multirow[b]{2}{*}{$\begin{array}{l}\text { Temp. } \\
(\mathrm{K})\end{array}$} & \multicolumn{5}{|c|}{ Total energy of supercell $(\mathrm{eV})^{\S}$} & \multicolumn{2}{|c|}{$\Delta \mathrm{E}(\mathrm{eV})^{\epsilon}$} \\
\hline & Ettringite & $\begin{array}{c}\text { Ettringite- } \\
\mathrm{SO}_{4-1}{ }^{\dagger}\end{array}$ & $\begin{array}{l}\text { Ettringite- } \\
\mathrm{SO}_{4}-2 \mathrm{a}^{\dagger}\end{array}$ & Gypsum & $\begin{array}{l}\text { Gypsum- } \\
\mathrm{SO}_{4}-\mathrm{H} 1^{\ddagger}\end{array}$ & $\begin{array}{c}\text { Based on } \\
\text { Ettringite- } \\
\mathrm{SO}_{4-1}\end{array}$ & $\begin{array}{c}\text { Based on } \\
\text { Ettringite- } \\
\mathrm{SO}_{4}-2 \mathrm{a}\end{array}$ \\
\hline 0 & -1354.778 & -1355.635 & -1355.318 & -1115.361 & -1115.556 & 0.66 & 0.35 \\
\hline 15 & -1354.013 & -1354.880 & -1354.762 & -1114.722 & -1114.946 & 0.64 & 0.53 \\
\hline 298 & -1344.289 & -1345.298 & -1345.449 & -1107.421 & -1107.673 & 0.76 & 0.91 \\
\hline
\end{tabular}

${ }^{\S}$ From ensemble averages calculated using the last 6 ps of the AIMD trajectories at $15 \mathrm{~K}$ and $298 \mathrm{~K}$. From final energy of energy minimization at $0 \mathrm{~K}$.

${ }^{\epsilon}$ Net Reaction: [Ettringite-SO $\left.{ }_{4}-1 / 2 \mathrm{a}\right]+\left[\right.$ Gypsum] $\rightarrow$ [Ettringite] $+\left[\right.$ Gypsum-SO $\left.{ }_{4}-\mathrm{H} 1\right]$

${ }^{\dagger}$ Configurations selected in the LCFs to the ettringite samples.

*Configuration Gypsum-SO $\mathrm{S}_{4}-\mathrm{H} 2$ is $0.053,0.055$, and $0.025 \mathrm{eV}$ higher in energy than configuration Gypsum-SO $\mathrm{SO}_{4} \mathrm{H} 1$ at 0,15 , and $298 \mathrm{~K}$, respectively. Using Gypsum-SO $\mathrm{SO}_{4}-\mathrm{H} 2$ instead of Gypsum-SO $\mathrm{S}_{4}-\mathrm{H} 1$ in these calculations will therefore make $\Delta \mathrm{E}$ slightly more positive in all cases. 


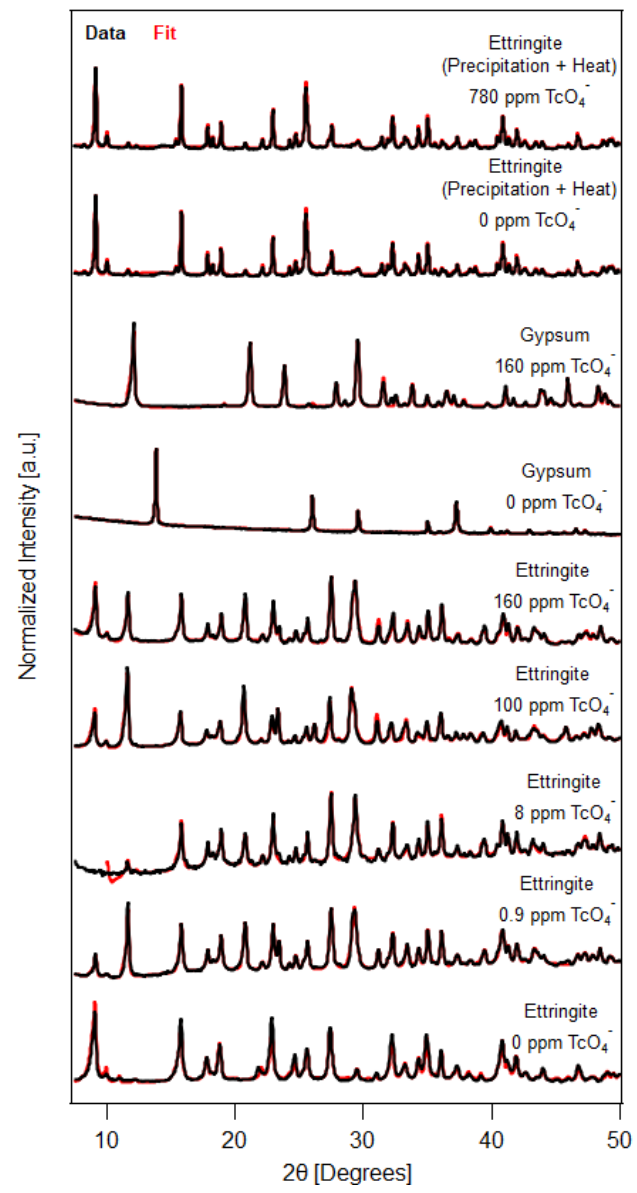

Figure S5. XRD patterns and corresponding fits for ettringite and gypsum solids containing up to $780 \mathrm{ppm} \mathrm{TcO}_{4}{ }^{-}$. 

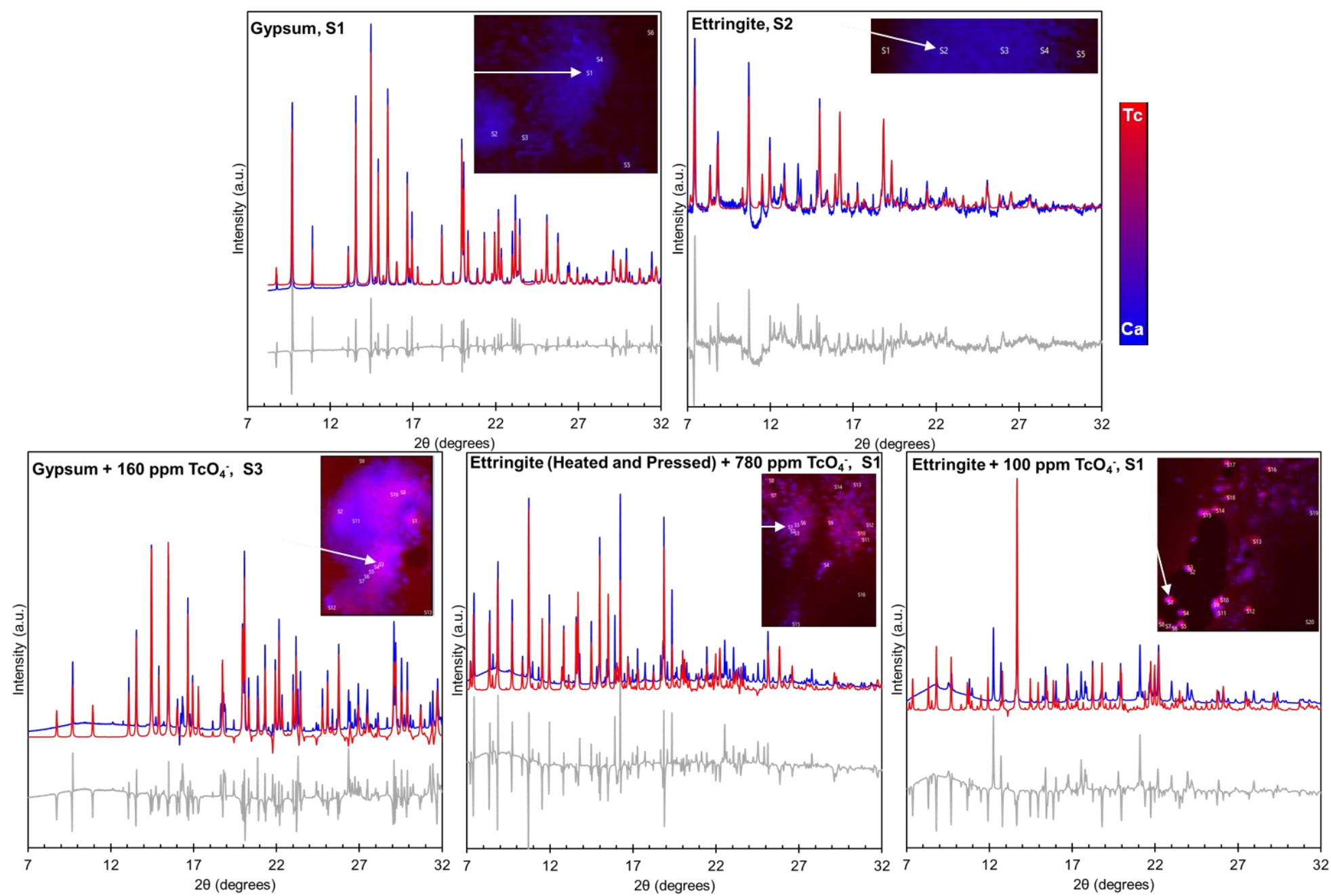

Figure S6. Microprobe HE-XRD patterns (red) of select spots identified in ettringite and gypsum solids containing between 0 and 780 ppm TcO$_{4}^{-}$. Rietveld fits (blue) and corresponding residuals (grey) are also provided. The selected patterns were determined to have the highest $R_{w p}$ value of all spots analyzed for the respective sample. Corresponding $\mu$ XRF maps that overlay Tc (red) and Ca (blue) are provided for each with the spots analyzed by HE-XRD identified. 
Table S5. Average Lattice Parameters and Mineralogical Distribution in Select Solids Determined by Microprobe HE-XRD*

\begin{tabular}{|c|c|c|c|c|c|c|}
\hline Parameter & Units & Ettringite (RP) & Gypsum (RP) & $\begin{array}{c}\text { Ettringite, } \\
100 \text { ppm TcO }_{4}^{-}(\mathrm{RP})\end{array}$ & $\begin{array}{c}\text { Gypsum, } \\
160 \text { ppm TcO }_{4}^{-}(\mathrm{RP})\end{array}$ & $\begin{array}{l}\text { Ettringite (Heat/Press), } \\
780 \text { ppm TcO4- (PP**) }\end{array}$ \\
\hline \multicolumn{7}{|c|}{ Ettringite $\left(\mathrm{P}_{3} / \mathrm{mmc}\right)$} \\
\hline$a$ & $\AA$ & $11.1857 \pm 0.0014$ & - & $11.226 \pm 0.001$ & - & $11.1883 \pm 0.0006$ \\
\hline$c$ & $\AA$ & $21.5224 \pm 0.0019$ & - & $21.5007 \pm 0.0013$ & - & $21.3575 \pm 0.0008$ \\
\hline$a: c$ & & $1: 1.924$ & - & 1:1.915 & - & 1:1.909 \\
\hline Volume & $\AA^{3}$ & $2332.12 \pm 0.26$ & - & $2346.57 \pm 0.26$ & - & $2315.3 \pm 0.13$ \\
\hline Fraction & $\%$ & 100 & - & 52.29 & - & 64.99 \\
\hline \multicolumn{7}{|c|}{ Gypsum $(C 2 / c)$} \\
\hline$a$ & $\AA$ & - & $6.274 \pm 0.0010$ & $6.2632 \pm 0.0063$ & $6.2724 \pm 0.0016$ & $6.2616 \pm 0.0031$ \\
\hline$b$ & $\AA$ & - & $15.1728 \pm 0.0004$ & $15.1646 \pm 0.0019$ & $15.1757 \pm 0.0006$ & $15.149 \pm 0.0012$ \\
\hline$c$ & $\AA$ & - & $5.6655 \pm 0.0008$ & $5.6599 \pm 0.0051$ & $5.6631 \pm 0.0014$ & $5.6484 \pm 0.0027$ \\
\hline$a: b: c$ & & - & 1.107:2.678: 1 & $1.107: 2.679: 1$ & 1.108:2.68: 1 & 1.109:2.682: 1 \\
\hline$\beta$ & $\circ$ & - & 114.0924 & 114.125 & 114.0972 & 114.148 \\
\hline Volume & $\AA 3$ & - & $492.32 \pm 0.01$ & $490.62 \pm 0.05$ & $492.09 \pm 0.02$ & $488.9 \pm 0.04$ \\
\hline Fraction & $\%$ & - & 100 & 23.52 & 100 & 23.49 \\
\hline \multicolumn{7}{|c|}{ Calcite $(R \overline{3 c})$} \\
\hline$a$ & $\AA$ & - & - & $4.9684 \pm 0.0007$ & - & $4.9742 \pm 0.0015$ \\
\hline$c$ & $\AA$ & - & - & $17.0995 \pm 0.0014$ & - & $17.0051 \pm 0.0030$ \\
\hline Volume & А3 & - & - & $365.53 \pm 0.04$ & - & $364.38 \pm 0.09$ \\
\hline Fraction & $\%$ & - & - & 24.18 & - & 11.5 \\
\hline $\mathrm{R}_{\mathrm{wp}}$ & $\%$ & 1.209 & 1.817 & 4.144 & 1.761 & 3.771 \\
\hline
\end{tabular}

* All lattice parameters and mineral fractions are average of the $100 \mu \mathrm{m}^{2}$ spots analyzed for each sample. The number of spots ranged from 5 to 19 . The $\mathrm{R}_{\mathrm{wp}}$ value is the median value. Sample powders were embedded in epoxy to form thin sections for analysis.

** Pressed pellet contacted with TcO4- was crushed to a powder before embedding in epoxy for $\mu \mathrm{XRF}$ and HE-XRD analysis. 

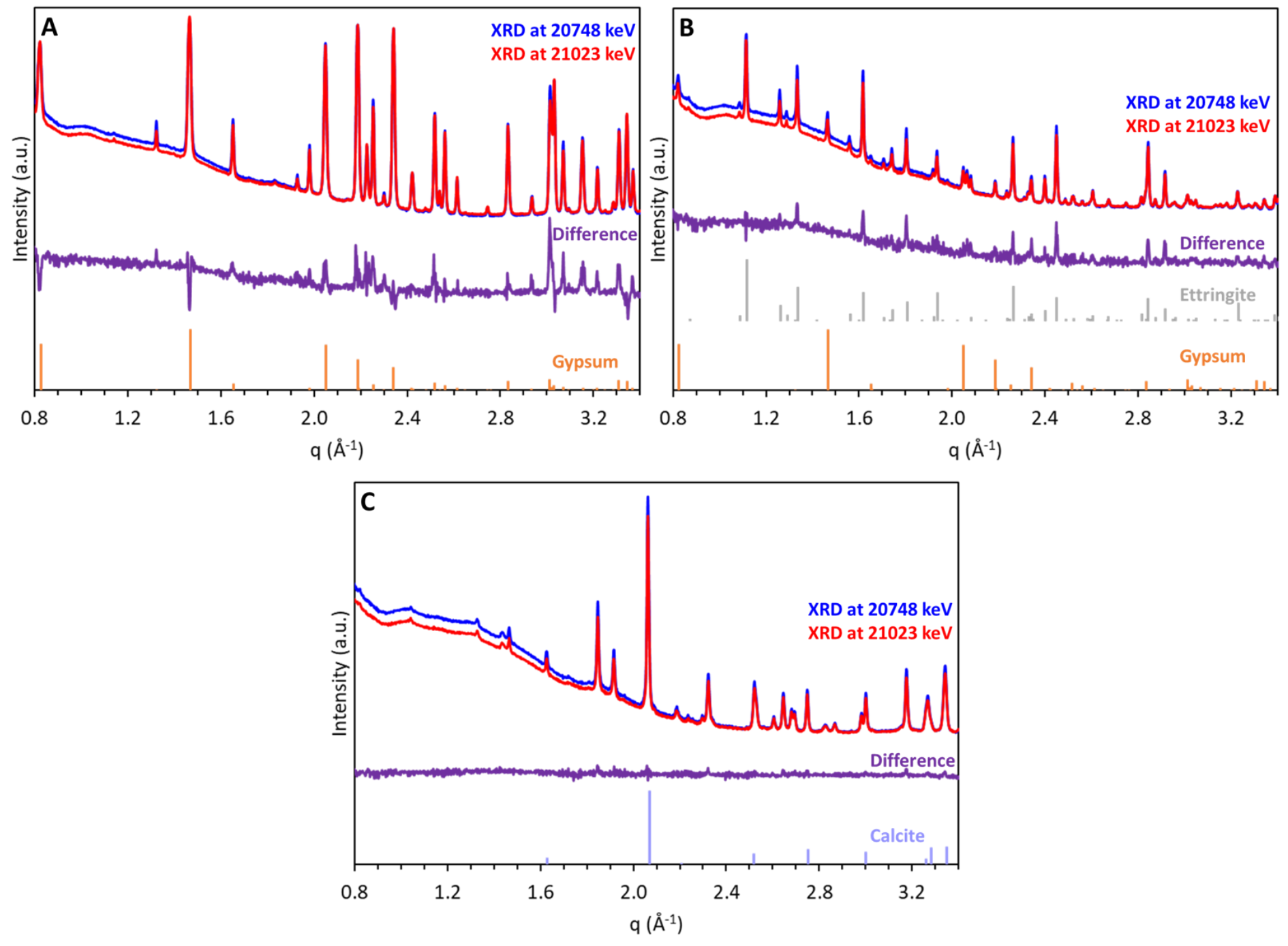

Figure S7. aXRD patterns identify ettringite as the preferred mineral phase for $\mathbf{T c O}_{4}{ }^{-}$incorporation. (A) gypsum precipitated in the presence of $160 \mathrm{ppm} \mathrm{TcO}_{4}^{-}$, (B) ettringite precipitated, heated, and pressed before contacting with $780 \mathrm{ppm} \mathrm{TcO}_{4}^{-}$in solution, and (C) an ettringite depleted area in the batch precipitated ettringite sample prepared in the presence of $\mathrm{TcO}_{4}{ }^{-}$. 


\section{References}

(1) Webb, S. In AIP Conference Proceedings; AIP: 2011; Vol. 1365, p 196.

(2) Kresse, G.; Hafner, J. Ab initio molecular dynamics for liquid metals. Physical Review B. 1993, 47, 558.

(3) Kresse, G.; Hafner, J. Ab initio molecular-dynamics simulation of the liquid-metal-amorphoussemiconductor transition germanium. Physical Review B. 1994, 49, 14251.

(4) Kresse, G.; Furthmuller, J. Efficient iterative schemes for ab initio total-energy calculations using a plane-wave basis set. Physical Review B. 1996, 54, 11169.

(5) Blöchl, P. E. Projector augmented-wave method. Physical Review B. 1994, 50, 17953.

(6) Kresse, G.; Joubert, D. From ultrasoft pseudopotentials to the projector augmented-wave method. Physical Review B. 1999, 59, 1758.

(7) Perdew, J. P.; Burke, K.; Ernzerhof, M. Generalized gradient approximation made simple. Physical Review Letters. 1996, 77, 3865.

(8) Perdew, J. P.; Burke, K.; Ernzerhof, M. Generalized gradient approximation made simple [Phys. Rev. Lett. 77, 3865 (1996)]. Physical Review Letters. 1997, 78, 1396.

(9) Grimme, S. Semiempirical GGA-type density functional constructed with a long-range dispersion correction. Journal of Computational Chemistry. 2006, 27, 1787.

(10) Hartman, M. P.; Berliner, R. Investigation of the structure of ettringite by time-of-flight neutron powder diffraction techniques. Cement and Concrete Research. 2006, 36, 364.

(11) Henry, P. F.; Weller, M. T.; Wilson, C. C. Neutron powder diffraction in materials with incoherent scattering: an illustration of Rietveld refinement quality from nondeuterated gypsum. J. Appl. Cryst. 2009, 42, 1176.

(12) German, K. E.; Grigoriev, M. S.; Garashchenko, B. L.; Kopytin, A. V.; Tyupina, E. A. Redetermination of the crystal structure of $\mathrm{NaTcO}_{4}$ at 100 and $296 \mathrm{~K}$ based on single-crystal Xray data. Act. Cryst. E. 2017, 73, 1037.

(13) Rehr, J. J.; Albers, R. C. Theoretical approaches to x-ray absorption fine structure. Rev. Mod. Phys. 2000, 72, 621.

(14) Rehr, J. J.; Kas, J. J.; Prange, M. P.; Sorini, A. P.; Takimoto, Y.; Vila, F. D. Ab initio theory and calculations of X-ray spectra. C.R. Phys. 2009, 10, 548.

(15) Rehr, J. J.; Kas, J. J.; Vila, F. D.; Prange, M. P.; Jorissen, K. Parameter-free calculations of X-ray spectra with FEFF9. Phys. Chem. Chem. Phys. 2010, 12, 5503.

(16) Newville, M. IFEFFIT: Interactive XAFS analysis and FEFF fitting. Journal of Synchrotron Radiation. 2001, 8, 322. 